\title{
Padecer una fractura de cadera aumenta el riesgo de muerte inmediato en mayores de 50 años
}

\author{
Hip fractures increase immediate death risk in individuals over 50s
}

\section{Objetivos}

Determinar la magnitud y duración del aumento de mortalidad luego de una fractura de cadera en adultos mayores.

\section{Fuente de datos}

Dos revisores independientes realizaron la búsqueda electrónica en MEDLINE y EMBASE de artículos publicados en cualquier idioma entre 1957 y mayo de 2009. También se realizó búsqueda manual de artículos de referencia.

\section{Selección de estudios}

Se priorizó la búsqueda de artículos de cohorte prospectivos que evaluaran la mortalidad a partir del momento de la fractura de cadera en hombres y mujeres mayores de 50 años. Se descartaron estudios no controlados, revisiones, estudios casos-control y aquellos estudios con menos de un año de seguimiento.

\section{Extracción de datos}

Dos revisores extrajeron en forma independiente datos de sobrevida y aquellos que se refieren a puntos relevantes para la calidad, validez y generalización de los resultados. Los desacuerdos se resolvieron por consenso.

\section{Resultados principales}

En los tres primeros meses de seguimiento luego de la fractura de cadera el riesgo relativo de morir por distintas causas fue de 5,75 (IC95\% 4,94 a 6,67) en mujeres y de 7,95 $(6,13$ a 10,3) en hombres.

El riesgo relativo disminuyó sustancialmente luego del periodo
Haentjens P y col. Ann Intern Med. 2010 Mar 16;152(6):380-90 de tres meses pero no retornó a valores normales cuando se comparó el grupo de fractura de cadera con un grupo control ajustado por edad y sexo (ver tabla 1 ).

Tabla 1: Riesgo relativo* de mortalidad por todas las causas en mujeres y hombres con fractura de cadera comparados con participantes controles en un intervalo de tiempo dado.

\begin{tabular}{l|c|c}
$\begin{array}{c}\text { Intervalo } \\
\text { de edad }\end{array}$ & $\begin{array}{c}\text { Mujeres } \\
\text { RR (IC95\%) }\end{array}$ & $\begin{array}{c}\text { Varones } \\
\text { RR (IC95\%) }\end{array}$ \\
\hline 0 a 3 meses & $5,75(4,94$ a 6,69$)$ & $7,95(6,13$ a 10,30 $)$ \\
\hline 3 a 6 meses & $3,32(2,18$ a 5,07$)$ & $3,56(2,64$ a 4,80$)$ \\
\hline 6 a 9 meses & $1,92(1,59$ a 2,32$)$ & $2,33(1,91$ a 2,85$)$ \\
\hline 9 a 12 meses & $1,59(1,26$ a 2,00$)$ & $2,30(1,81$ a 2,93$)$ \\
\hline 0 a 1 año & $2,87(2,52$ a 3,27$)$ & $3,70(3,31$ a 4,14) \\
\hline 1 a 2 años & $1,86(1,60$ a 2,16$)$ & $1,90(1,58$ a 2,30$)$ \\
\hline 2 a 3 años & $1,58(1,09$ a 2,29$)$ & $1,69(1,36$ a 2,10$)$ \\
\hline
\end{tabular}

RR: riesgo relativo

\section{Conclusiones}

El riesgo de morir posteriormente a una fractura de cadera se encuentra aumentado tanto en hombres como en mujeres en los tres meses subsiguientes al evento. Este riesgo disminuye pasados estos primeros tres meses pero no retorna a valores iguales a los previos al evento.

Palabras claves: fractura de cadera, mortalidad, ancianos. Key words: hip fracture, mortality, older patients.

Fuente de financiamiento: Fund for Scientific Research and Willy Gepts Foundation, Universitair Ziekenhuis Brussel

\section{Comentario}

Las fracturas del fémur (proximal) se asocian a dependencia, invalidez y mortalidad ${ }^{1}$. Se producen a nivel mundial aproximádamente 1,6 millones de fracturas de cadera por año. Se realizaron proyecciones que estiman para América Latina un incremento en el número de fracturas de cadera de $700 \%$ para los mayores de 65 años entre los años 1990 al 2050². En Argentina la incidencia de fractura de cadera es de 182 a 304 fracturas por cada 100.000 habitantes mayores de 50 años por año $0^{3,4}$

Se ha descrito una tasa de mortalidad de hasta un 25 a $30 \%$ al año del evento. Los pacientes que sobreviven presentan diferentes complicaciones: aproximadamente el $40 \%$ camina con ayuda mecánica, el $60 \%$ requiere asistencia y el $33 \%$ necesita internación en hogares o se tornan totalmente dependientes; todo esto genera graves e importantes implicancias para el sistema de salud.

Dado el aumento en la expectativa de vida como el mayor riesgo de fractura de cadera con la edad y las graves consecuencias que esto acarrea para la población deben enfocarse los esfuerzos en tareas de prevención de la misma.

*Ver glosario

Fabiana Giber [ Servicio de Clínica Médica del Hospital Italiano de Buenos Aires fabiana.giber@ hospitalitaliano.org.ar ]

Giber F. Padecer una fractura de cadera aumenta el riesgo de muerte inmediato en mayores de 50 años. Evid Act Práct Ambul Jul-Set $2011 ; 14(3): 95$. Comentado de: Haentjens P, Magaziner J, Colón-Emeric CS y col. Meta-analysis: excess mortality after hip fracture among older women and men. Ann Intern Med. 2010 Mar 16;152(6):380-90. PMID: 20231569.

\section{Referencias}

1. Melton LJ y col. Adverse outcomes of osteoporotic fractures in the general population. J Bone Miner Res. 2003;18(6): 1139-41.

1. Melton $\mathrm{LJ}$ y col. Adverse outcomes of osteoporotic fractures in the general population. J Bone Min

3. Bagur A y col. Epidemiología de las fracturas del fémur proximal en La Plata, Argentina. Medicina 1991;51:343-7.

4. Morosano M y col. Incidence of hip fracture in the city of Rosario, Argentina. Osteoporosis Int 2005;16(11):1339-44. 